\title{
Identificación y evaluación de riesgos posturales en un proceso de acabado de piezas automotrices
}

\author{
Identification and Evaluation of Postural Risks \\ in a Process of Finishing Automotive Parts \\ Identificação e avaliação de riscos posturais \\ em um processo de acabamento de peças \\ automotivas
}

Luis Cuautle Gutiérrez, PhD1*

Luis Alberto Uribe Pacheco, M. S. ${ }^{1}$

José Domingo García Tepox, M. S. ${ }^{1}$

Recibido: 26 de noviembre de $2019 \cdot$ Aceptado: 26 de noviembre de 2020

Doi: https://doi.org/10.12804/revistas.urosario.edu.co/revsalud/a.10053

Para citar este artículo: Cuautle Gutiérrez L, Uribe Pachecho LA, García Tepox JD. Identificación y evaluación de riesgos posturales en un proceso de acabado de piezas automotrices. Rev Cienc Salud. 2021;19(1):1-14.

https://doi.org/10.12804/revistas.urosario.edu.co/revsalud/a.10053

\section{Resumen}

Introducción: actualmente, los procesos automáticos predominan en el sector automotor, así como en otros giros industriales. De esta manera, la participación humana se restringe a la carga y descarga de materiales. La Organización Internacional de Estandarización ha desarrollado normas para evaluar posturas de trabajo estáticas; mientras que la ergonomía emplea metodologías y herramientas para evaluar riesgos posturales. El objetivo fue identificar, evaluar y reducir los riesgos posturales a los que están expuestos los trabajadores de una operación de acabado de piezas automotrices en un proceso de inyección de plástico. Materiales y métodos: se analizaron las condiciones existentes de una estación de trabajo respecto a la carga y descarga de materiales con métodos ergonómicos, como la evaluación rápida de todo el cuerpo, el sistema de análisis de trabajo Ovako, la ecuación de carga del Instituto Nacional para la Seguridad y Salud Ocupacional y el método de indicadores clave para operaciones de manejo manual. Además, se diseñó un nuevo dispositivo que permitiera el traslado de los materiales a través de la estación de trabajo.

1 Facultad de Ingeniería Industrial y Automotriz, Universidad Popular Autónoma del Estado de Puebla, Puebla (México).

Luis Cuautle: oRCID https://orcid.org/0000-0003-2424-2381

Luis Alberto Uribe Pacheco: orCID https://orcid.org/0000-0002-1804-044

José Domingo García Tepox: ORCiD https://orcid.org/0000-0001-7030-7735

Autor de correspondencia: luis.cuautle@upaep.mx 
Resultados: los resultados ergonómicos obtenidos muestran índices muy altos que ponen en riesgo a los trabajadores estudiados. La implementación de la mejora sugerida evita la carga por parte del personal y con ello se reducen los factores de lesiones músculo-esqueléticas. Conclusión: la combinación de diversos estudios ergonómicos contribuye a evaluar las posturas de trabajo y a reducir los esfuerzos de carga y la probabilidad de sufrir una lesión.

Palabras clave: ergonomía; riesgos posturales; sector automotor.

\section{Abstract}

Introduction: Currently, automatic processes predominate in the automotive sector, as well as in other industrial areas. In this way, human participation is restricted to the loading and unloading of materials. The international standardization organization has developed standards for the evaluation of static work postures while ergonomics employs methodologies and tools for the evaluation of postural risks. The objective was to identify, evaluate and reduce the postural risks to which the workers of a finishing operation of automotive parts in a plastic injection process are exposed. Materials and methods: The existing conditions of a work station regarding the loading and unloading of materials through ergonomic methods such as the rapid entire body assessment, the Ovako work analysis system, the load equation of the National Institute for Occupational Safety and Health and the method of key indicators for manual handling operations. In addition, a new device was designed to allow the transfer of materials through the workstation. Results: The ergonomic results obtained show very high levels of risk that put at risk the workers studied. The implementation of the suggested improvement avoids the load on the part of the personnel and with it reduces the factors of musculoskeletal injuries. Conclusions: The combination of several ergonomic studies contributes to evaluate the work postures and reduce the loading efforts and the probability of suffering an injury.

Keywords: Ergonomics; postural risk; automotive sector.

\section{Resumo}

Introdução: atualmente, os processos automáticos predominam no setor automotivo, bem como em outros setores industriais. Desta forma, a participação humana fica restrita ao carregamento e descarregamento de materiais. A organização internacional de padronização desenvolveu padrões para a avaliação de posturas estáticas de trabalho, enquanto a ergonomia usa metodologias e ferramentas para a avaliação de riscos posturais. O objetivo foi identificar, avaliar e reduzir os riscos posturais a que estão expostos os trabalhadores de uma operação de acabamento de peças automotivas em um processo de injeção plástica. Materiais e métodos: as condições existentes de uma estação de trabalho foram avaliadas quanto ao carregamento e descarregamento de materiais através de métodos ergonômicos, tais como a avaliação rápida de corpo inteiro, o sistema de análise de trabalho Ovako, a equação de carga do Instituto Nacional de Segurança e Saúde Ocupacional e o método de indicadores chaves para operações de controle manual. Além disso, um novo dispositivo foi projetado para permitir a transferência de materiais ao longo da estação de trabalho. Resultados: os resultados ergonômicos obtidos mostram níveis de risco muito elevados que colocam em risco os trabalhadores avaliados. A implementação da melhoria sugerida evita sobrecarga da equipe e, portanto, reduz os fatores de lesões musculoesqueléticas. Conclusão: a combinação de diversos estudos ergonômicos contribui para avaliar as posturas de trabalho e reduzir os esforços de carga e a probabilidade de sofrer uma lesão.

Palavras chave: ergonomia; riscos posturais; setor automotivo. 


\section{Introducción}

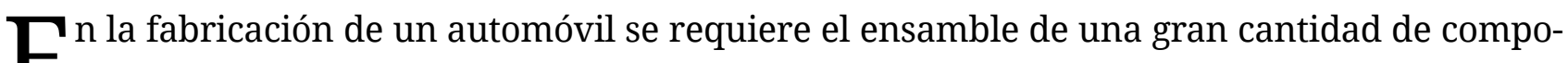
Enentes, mismos que son fabricados en diversos procesos de manufactura. Tradicionalmente, los procesos son automáticos lo que implica que la participación humana sea solo en actividades de carga y descarga de materiales, situación que pone en riesgo la integridad física de los trabajadores (1). Sin embargo, por el tamaño, el peso y las características del vehículo, los componentes llegan a ser difíciles de manipular por una o dos personas.

Por su parte, la Asociación Española de Ergonomía establece que la ergonomía es un conjunto de conocimientos multidisciplinarios aplicados al diseño de los productos y el diseño de los puestos de trabajo (2). En este segundo aspecto, la gran cantidad de factores de riesgo considerados exige la elección de un método de evaluación ergonómica para analizar movimientos repetitivos, levantamiento de cargas, posturas estáticas y forzadas, así como exigencias mentales y monotonía; de ahí la necesidad de aplicar una combinación de estos en una tarea específica. La tabla 1 muestra el método de evaluación ergonómica empleado en esta investigación, así como su alcance de aplicación correspondiente.

Tabla 1. Métodos de evaluación ergonómica

\begin{tabular}{|c|c|}
\hline Método de evaluación ergonómica & Alcance \\
\hline Evaluación rápida de todo el cuerpo & $\begin{array}{l}\text { Valoración rápida de las posturas del trabajador en } \\
\text { su puesto de trabajo }\end{array}$ \\
\hline Evaluación del riesgo postural ocupacional (REBA) & $\begin{array}{l}\text { Evaluación de posturas forzadas tanto estáticas como } \\
\text { dinámicas en ciclos cortos y repetitivos }\end{array}$ \\
\hline Sistema de análisis de trabajo Ovako (owAs) & $\begin{array}{l}\text { Observación, registro y análisis de carga postural. } \\
\text { Aplicable al sector industrial }\end{array}$ \\
\hline $\begin{array}{l}\text { Ecuación de carga del Instituto Nacional para la Se- } \\
\text { guridad y Salud Ocupacional (NIOSH) }\end{array}$ & $\begin{array}{l}\text { Evaluación de los riesgos ergonómicos relacionados } \\
\text { con el levantamiento manual de cargas y su trans- } \\
\text { porte }\end{array}$ \\
\hline $\begin{array}{l}\text { Método de indicadores clave para operaciones de } \\
\text { manejo manual (кIM-мно) }\end{array}$ & $\begin{array}{l}\text { Evaluación de riesgos en tareas de levantar, mante- } \\
\text { ner y llevar }\end{array}$ \\
\hline
\end{tabular}

En la identificación de aspectos ergonómicos se utilizan cuestionarios, entrevistas y registros visuales (3). Para la evaluación del riesgo de manejo manual tradicionalmente se han empleado tablas de Snook y Ciriello, cuadros de evaluación de manejo manual, la ecuación de levantamiento revisada del Instituto Nacional para la Seguridad y Salud Ocupacional (NIOSH), entre otros (4). Al respecto, la Organización Internacional de Estandarización (Iso, por sus siglas en inglés) cuenta con normas para la evaluación de posturas de trabajo estáticas y el grupo de normas iso11228 en sus volúmenes 1 y 2 señala los límites recomendados para los cinco movimientos fundamentales en el manejo manual de cargas (5-7). 
El Rapid Entire Body Assessment (REBA, por sus siglas en inglés) divide en dos grupos diferentes segmentos del cuerpo (8). El primer grupo abarca el cuello, el torso y las piernas; mientras que el segundo considera el brazo, el antebrazo y la muñeca. A través de la observación de la tarea estudiada, se otorga un puntaje a cada segmento del cuerpo según la postura tomada y con el uso de tablas numéricas se obtienen dos puntajes diferentes que representan el grado de carga postural músculo-esquelético. Las puntuaciones A y B se obtienen al agregar al puntaje anterior las consideraciones de agarre, carga y fuerza. Finalmente, con una tercera tabla se suma el puntaje $\mathrm{C}$, que se agrega a la cantidad anterior y se obtiene la calificación final o puntaje máximo. Esta última cantidad se encuentra dentro de un rango del 1 al 15 y se compara contra cinco niveles de acción y cinco niveles de riesgo previamente definidos.

Otro método que depende de la observación de las posturas adoptadas por el trabajador al ejecutar la tarea resulta ser el sistema de análisis de trabajo Ovako (owAs, por sus siglas en inglés). En este sistema es posible identificar hasta 252 posiciones diferentes resultados de la combinación de la posición de espalda (4 posiciones), brazos (3 posiciones), piernas (7 posiciones) y cargas levantadas (3 intervalos). Para la determinación del riesgo para el trabajador que presenta una postura, existen 4 niveles ascendentes: el valor 1 es el de menor riesgo y el valor de 4 es el que tiene un mayor riesgo. Este método se aplicó a la evaluación ergonómica de un puesto de trabajo del sector metalmecánico (9).

En cuanto a los riesgos asociados por el manejo manual de material con carga se emplea la ecuación de carga de Niosh, que recomienda el peso máximo aceptable que la mayor parte de los trabajadores puede cargar en una jornada de 8 horas sin aumentar el riesgo de trastornos músculo-esqueléticos.

En el 2007, el Instituto Federal Alemán de Seguridad y Salud Ocupacional creó el método indicador clave para operaciones de manejo manual (кIм-мно, por sus siglas en inglés), con el propósito de evaluar tareas que involucran la exposición del área dedo-mano-brazo cuando se trabaja con objetos físicos. Las repeticiones frecuentes de operaciones manuales similares, así como operaciones de destreza o de reconocimiento de pequeños detalles son ejemplos típicos de este indicador (10).

\section{Materiales y métodos}

Ll proceso que se estudió consiste en el acabado de un guarda fango en una empresa de inyecEción de plásticos que genera componentes para el sector automotor, localizada en Puebla (México). Dicho proceso abarca las operaciones de eliminado de rebabas, inspección de calidad, etiquetado y colocación de eliminadores de ruido. Para su traslado se acumulan 5 componentes y se depositan en una caja (el total de esta carga es igual a 8 kilogramos). La actividad la realizan tres personas, generalmente del género femenino, por lo que el volumen y peso de 
los cinco componentes genera deterioro del producto y problemas en la espalda. Debido a esto último, el objetivo de la presente investigación es identificar y evaluar los riesgos posturales a los que están expuestas las trabajadoras.

Los métodos ergonómicos empleados para tal fin fueron: REBA, owAs, NIOSH y KIM. Adicionalmente, con ayuda del software DELMIA se realizó un análisis del consumo de kilocalorías. A partir de los hallazgos, se plantea una propuesta de solución con el objeto de reducir el riesgo de lesión en este proceso, así como una disminución en el consumo energético por parte de las personas que realizan la actividad.

\section{Análisis de operaciones con el método REBA}

Durante dos meses se observó y se tomaron videos de la actividad en cuestión. A partir de ello, se construyeron diagramas de flujo a efectos de dividir en elementos más detallados la tarea y determinar los riesgos ergonómicos presentes. Siguiendo el protocolo de la REBA, para la actividad de sujetar los cinco componentes, se determinó un puntaje A igual a 7 más un punto por cuestiones de carga y fuerza. Respecto a la tabla B, el puntaje obtenido fue de 5 más 3 puntos debido al agarre. Finalmente, a través de la tabla $C$ se dio una calificación final de 11 puntos. El detalle del análisis de muestra en la figura 1.

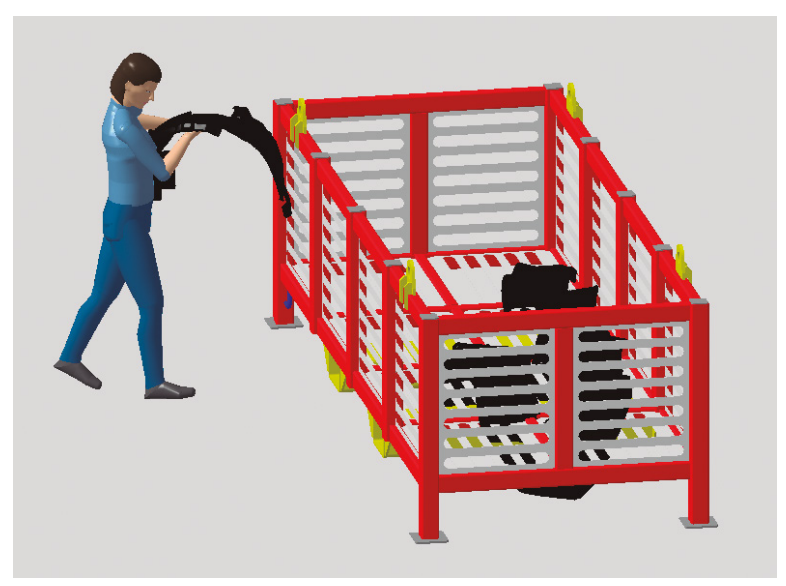

\section{Formato REBA}

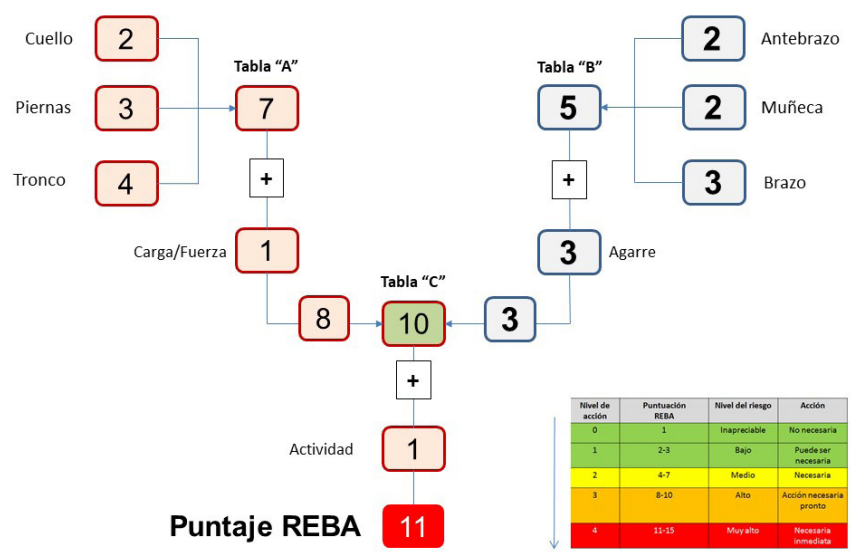

Figura 1. Análisis REBA de la actividad de sujeción de componentes

Para la actividad de colocar los cinco componentes en la caja, se hizo el mismo análisis y se determinó una calificación REBA de 12 puntos. En la figura 2 se muestran los puntajes otorgados del análisis correspondiente. 

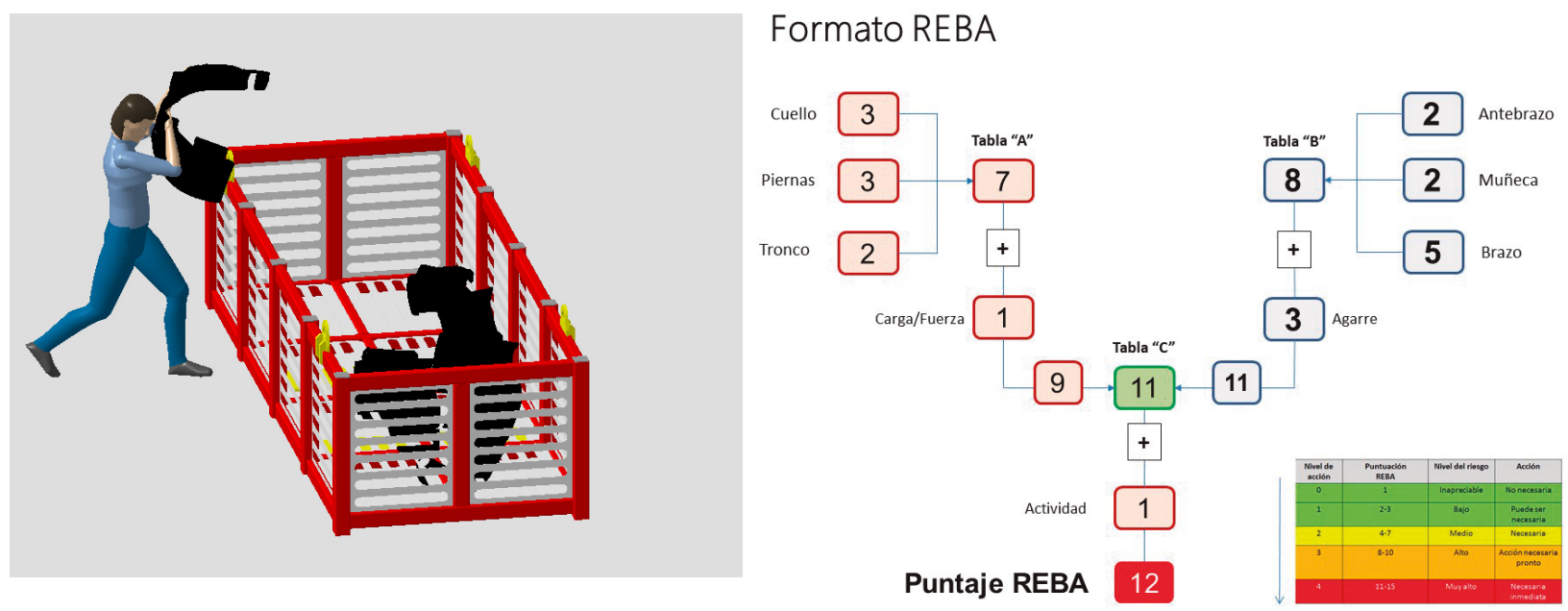

Figura 2. Análisis REBA de la actividad de colocación de componentes

Finalmente, para la actividad de acomodo de componentes en caja se realizó el mismo análisis y el detalle de puntuación se presenta en la figura 3. Se hace mención de la postura adoptada por la operadora en términos de la espalda y la calificación de 12 puntos según la REBA.

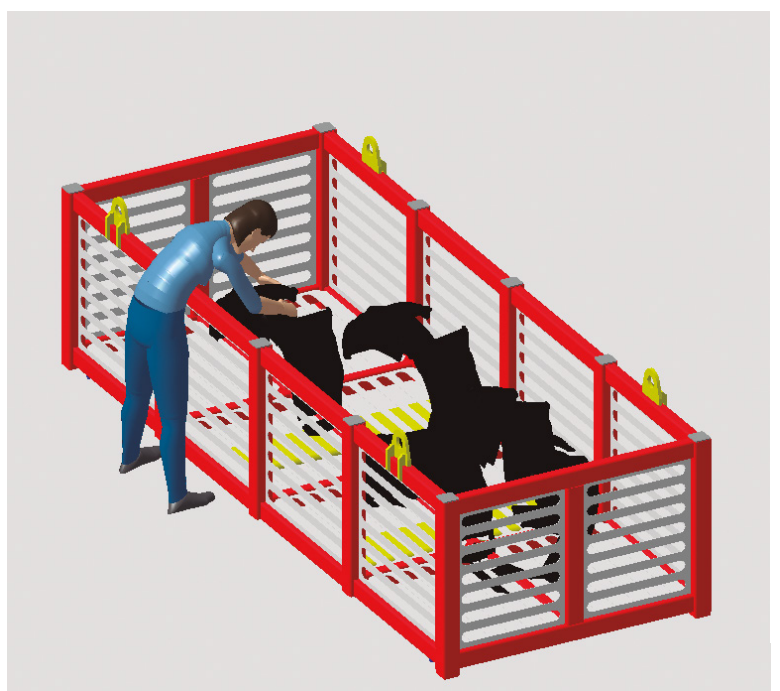

\section{Formato REBA}

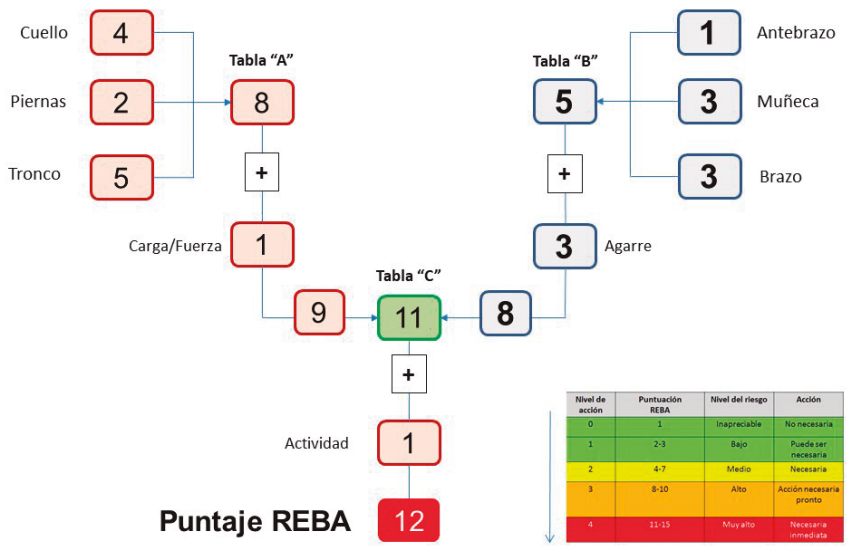

Figura 3. Análisis REBA de la actividad de acomodo de componentes

\section{Análisis de operaciones con el método owAS}

Para confirmar la información obtenida del análisis REBA, se realizó un owAs de las tres actividades estudiadas. La tabla 2 señala el estudio hecho a la actividad de sujeción de componentes. Las calificaciones otorgadas fueron para la espalda 4, brazos 1, piernas 5 y por cuestiones de fuerza peso 1. De tal forma que la categoría de acción fue de 4, lo que conlleva la aplicación de acciones correctivas inmediatas. 
Tabla 2. Análisis owAs de la actividad de sujeción de componentes

\begin{tabular}{|c|c|c|c|c|c|c|c|c|c|c|c|c|c|c|c|c|c|c|c|c|c|c|c|c|}
\hline \multirow{3}{*}{ ESPALDA } & \multirow{3}{*}{4} & & & \multicolumn{21}{|c|}{ Piernas } \\
\hline & & \multicolumn{2}{|c|}{ Carga/Fuerza } & \multicolumn{3}{|c|}{1} & \multicolumn{3}{|c|}{2} & \multicolumn{3}{|c|}{3} & \multicolumn{3}{|c|}{4} & \multicolumn{3}{|c|}{5} & \multicolumn{3}{|c|}{6} & \multicolumn{3}{|c|}{7} \\
\hline & & Espalda & Brazos & 1 & 2 & 3 & 1 & 2 & 3 & 1 & 2 & 3 & 1 & 2 & 3 & 1 & 2 & 3 & 1 & 2 & 3 & 1 & 2 & 3 \\
\hline \multirow{3}{*}{ BRAZOS } & \multirow{3}{*}{1} & \multirow{3}{*}{1} & 1 & 1 & 1 & 1 & 1 & 1 & 1 & 1 & 1 & 1 & 2 & 2 & 2 & 2 & 2 & 2 & 1 & 1 & 1 & 1 & 1 & 1 \\
\hline & & & 2 & 1 & 1 & 1 & 1 & 1 & 1 & 1 & 1 & 1 & 2 & 2 & 2 & 2 & 2 & 2 & 1 & 1 & 1 & 1 & 1 & 1 \\
\hline & & & 3 & 1 & 1 & 1 & 1 & 1 & 1 & 1 & 1 & 1 & 2 & 2 & 2 & 2 & 2 & 3 & 1 & 1 & 1 & 1 & 1 & 2 \\
\hline \multirow{3}{*}{ PIERNAS } & \multirow{3}{*}{5} & \multirow{3}{*}{2} & 1 & 2 & 2 & 3 & 2 & 2 & 3 & 2 & 2 & 3 & 3 & 3 & 3 & 3 & 3 & 3 & 2 & 2 & 2 & 2 & 3 & 3 \\
\hline & & & 2 & 2 & 2 & 3 & 2 & 2 & 3 & 2 & 2 & 3 & 3 & 4 & 4 & 3 & 4 & 4 & 3 & 3 & 4 & 2 & 3 & 4 \\
\hline & & & 3 & 3 & 3 & 4 & 2 & 2 & 3 & 3 & 3 & 3 & 3 & 4 & 4 & 4 & 4 & 4 & 4 & 4 & 4 & 2 & 3 & 4 \\
\hline \multirow{6}{*}{$\begin{array}{l}\text { FUERZA/ } \\
\text { PESO }\end{array}$} & \multirow{6}{*}{1} & \multirow{3}{*}{3} & 1 & 1 & 1 & 1 & 1 & 1 & 1 & 1 & 1 & 2 & 3 & 3 & 3 & 4 & 4 & 4 & 1 & 1 & 1 & 1 & 1 & 1 \\
\hline & & & 2 & 2 & 2 & 3 & 1 & 1 & 1 & 1 & 1 & 2 & 4 & 4 & 4 & 4 & 4 & 4 & 3 & 3 & 3 & 1 & 1 & 1 \\
\hline & & & 3 & 2 & 2 & 3 & 1 & 1 & 1 & 2 & 3 & 3 & 4 & 4 & 4 & 4 & 4 & 4 & 4 & 4 & 4 & 1 & 1 & 1 \\
\hline & & \multirow{3}{*}{4} & 1 & 2 & 3 & 3 & 2 & 2 & 3 & 2 & 2 & 3 & 4 & 4 & 4 & 4 & 4 & 4 & 4 & 4 & 4 & 2 & 3 & 4 \\
\hline & & & 2 & 3 & 3 & 4 & 2 & 3 & 4 & 3 & 3 & 4 & 4 & 4 & 4 & 4 & 4 & 4 & 4 & 4 & 4 & 2 & 3 & 4 \\
\hline & & & 3 & 4 & 4 & 4 & 2 & 3 & 4 & 3 & 3 & 4 & 4 & 4 & 4 & 4 & 4 & 4 & 4 & 4 & 4 & 2 & 3 & 4 \\
\hline
\end{tabular}

\begin{tabular}{|c|c|c|}
\hline $\begin{array}{l}\text { Categoría } \\
\text { de acción }\end{array}$ & Descripción & Acción \\
\hline 1 & $\begin{array}{c}\text { Postura mormal y natural sin efectos dañinos en el sistema } \\
\text { músculo-esquelético }\end{array}$ & No requiere de acción \\
\hline 2 & $\begin{array}{c}\text { Postura con posibilidad de causar daño al sistema } \\
\text { músculo-esquelético }\end{array}$ & $\begin{array}{l}\text { Se requieren acciones correctivas en un } \\
\text { futuro cercano }\end{array}$ \\
\hline 3 & $\begin{array}{c}\text { Postura con efectos dañinos sobre el sistema músculo- } \\
\text { esquelético }\end{array}$ & $\begin{array}{l}\text { Se requieren acciones correctivas lo antes } \\
\text { posible }\end{array}$ \\
\hline 4 & $\begin{array}{l}\text { La carga causada por esta postura tiene efectos } \\
\text { sumamente dañinos sobre el sistema músculo-esquelético }\end{array}$ & $\begin{array}{l}\text { Se requiere tomar acciones correctivas } \\
\text { inmediatamente }\end{array}$ \\
\hline
\end{tabular}

Los hallazgos encontrados para las dos actividades restantes se resumen en la tabla 3, donde la categoría de acción para la actividad de colocación de componentes es igual a 2; mientras que la actividad de acomodo presentó una categoría de acción 4.

Tabla 3. Hallazgos del análisis owAS

\begin{tabular}{lclc}
\hline \multicolumn{2}{c}{ Colocación de componentes } & \multicolumn{2}{c}{ Acomodo de componentes } \\
\hline \multicolumn{1}{c}{ Segmento del cuerpo } & Calificación & Segmento del cuerpo & Calificación \\
\hline Espalda & 1 & Espalda & 4 \\
Brazos & 3 & Brazos & 1 \\
Piernas & 4 & Piernas & 5 \\
Fuerza/peso & 1 & Fuerza/peso & 1 \\
Categoría de acción & 2 & Categoría de acción & 4 \\
\hline
\end{tabular}




\section{Análisis de operaciones con el método NIOSH}

Por medio del registro de video se observó que la carga recorre una distancia dentro de la estación de trabajo, por lo que es pertinente emplear la ecuación de levantamiento revisada de NIosh, a fin de determinar la carga máxima permisible. La tabla 4 muestra el análisis. Como se puede observar, el estudio en el origen obtiene un índice de carga igual a 1.47. Ello indica que el trabajo puede ocasionar problemas a algunos trabajadores y que es necesario el análisis a fondo de la actividad y plantear cambios. En el destino, el índice obtenido es igual a 0.85 , lo que implica que el trabajo lo pueden ejecutar la mayoría de los trabajadores.

Tabla 4. Análisis owAs de la actividad de sujeción de componentes

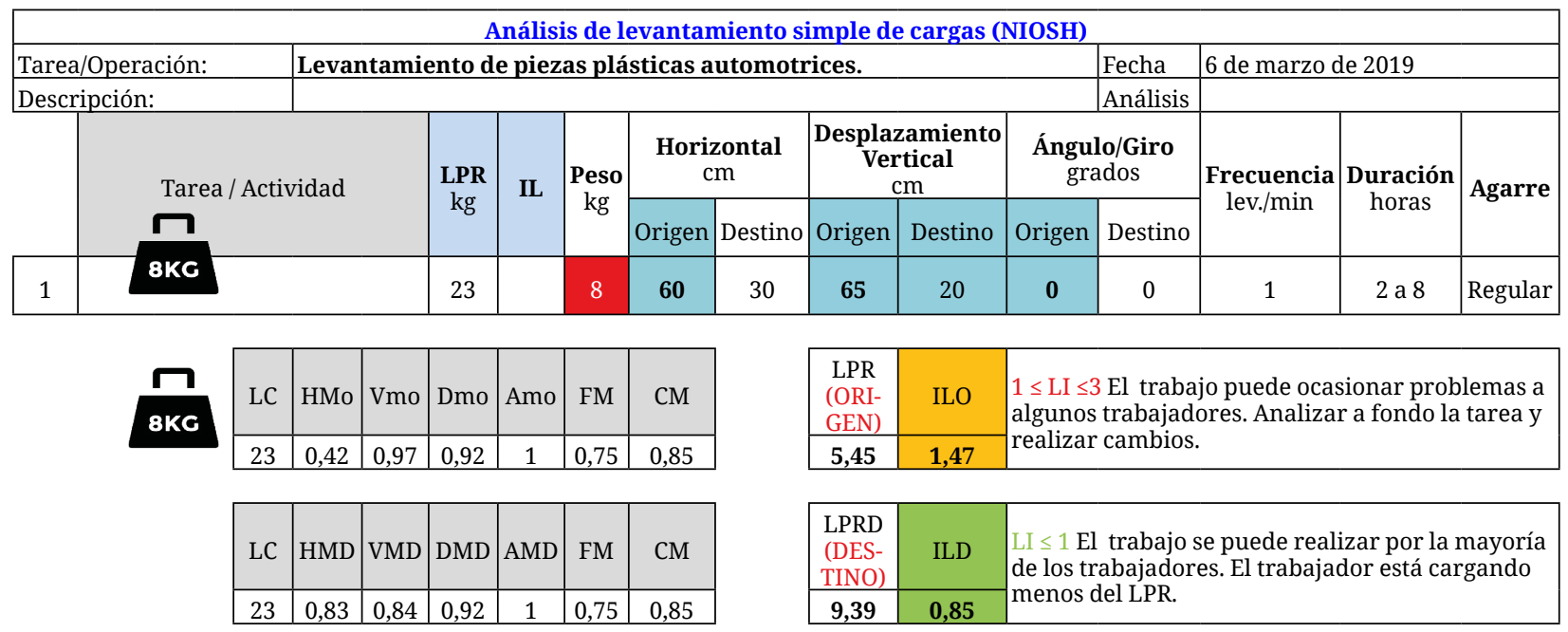

\section{Análisis de operaciones con el método KIM}

Al considerar que el proceso de acabado presenta carga y una postura de trabajo durante un tiempo determinado en unas condiciones de trabajo, se tomó la decisión de realizar un análisis empleando el método кıм. En la tabla 5 se exhibe el desglose del cálculo de puntuación de riesgo del proceso de acabado. El puntaje de riesgo obtenido fue 56, debido a la puntuación de la postura y del tiempo primordialmente. 
Tabla 5. Análisis Kim del proceso de acabado

Paso 3: Evaluación

La puntuación de la tarea o actividad deben introducirse y calcularse en el diagrama:

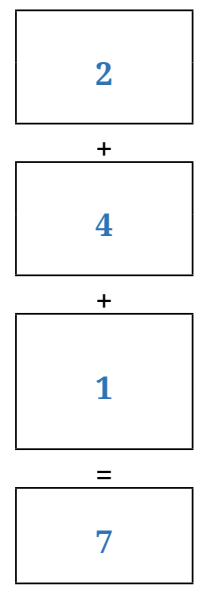

Puntuación de la Carga/Peso

Puntuación de la Postura

Puntuación de Condiciones de Trabajo

\begin{tabular}{|c|c|c|}
\hline Nivel de riesgo & Puntuación del riesgo & Descripción \\
\hline 1 & $<10$ & $\begin{array}{l}\text { Situación de baja carga, es poco probable que se } \\
\text { produzca una sobrecarga física. }\end{array}$ \\
\hline 2 & De $10 a<25$ & $\begin{array}{l}\text { Se trata de una situación de aumento de la carga, } \\
\text { es posible que se produzca una sobrecarga física } \\
\text { en los trabajadores menos "resistentes”, para este } \\
\text { grupo de personas, ayudaría volver a diseño de un } \\
\text { nuevo puesto de trabajo. }\end{array}$ \\
\hline 3 & De $25 a<50$ & $\begin{array}{l}\text { Es una situación de gran aumento de carga, y es } \\
\text { posible que se produzca una sobrecarga física } \\
\text { también para los trabajadores con una resistencia } \\
\text { "normal”. } \\
\text { Se recomienda volver a diseñar la estación de } \\
\text { trabajo. }\end{array}$ \\
\hline 4 & $\geq 50$ & $\begin{array}{l}\text { Es una situación de carga alta, es probable que se } \\
\text { produzca una sobrecarga física. } \\
\text { Es necesario entonces volver a diseñar la estación/ } \\
\text { puesto de trabajo. }\end{array}$ \\
\hline
\end{tabular}

\section{Determinación del consumo energético}

Finalmente, con ayuda del software DELMIA se simuló la tarea estudiada y se calculó el consumo energético por parte de las trabajadoras. El total de energía consumida es de $1142 \mathrm{kcal}$ en un tiempo de 23.177 s en una distancia de $9943.52 \mathrm{~mm}$, es decir, una tasa promedio de $2.94 \mathrm{kcal} / \mathrm{min}$.

\section{Propuesta de mejora}

Los estudios realizados con REBA muestran que las tres actividades que integran el proceso de acabado presentan un riesgo muy alto, por lo que se deben tomar acciones inmediatas. El análisis owAs señala que la carga causada por las posturas de trabajo de las actividades de sujeción y acomodo de componentes genera efectos dañinos sobre el sistema músculo-esquelético. Por último, el método кім confirma una sobrecarga física y plantea el rediseño del puesto de trabajo. 
De esta manera, se propone un dispositivo que acumule los cinco guardafangos y que no obligue a la trabajadora a cargarlos para su manipulación, sino que ahora jale dicha carga para su colocación en la caja de empaque. Con el uso de diseño asistido por computador se creó la propuesta y la figura 4 ilustra el concepto planteado.
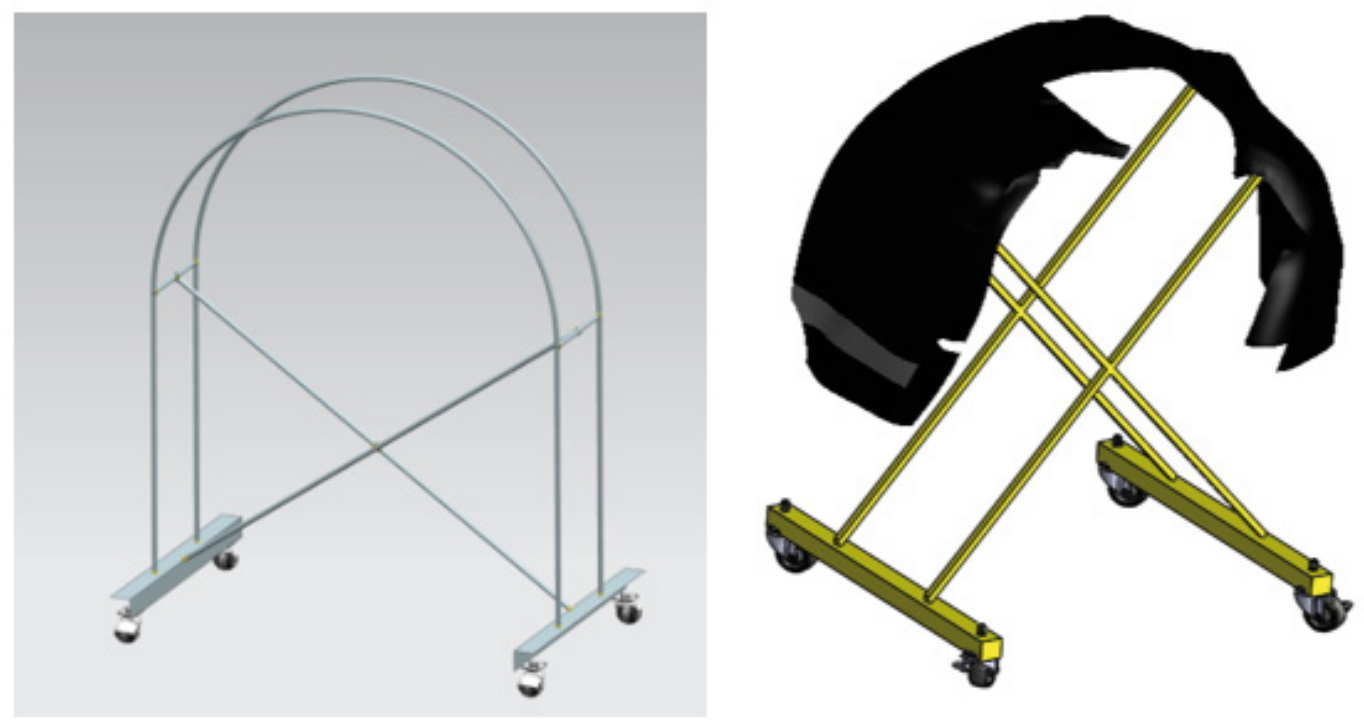

Figura 4. Dispositivo propuesto para el proceso de acabado

\section{Resultados}

C construyó el dispositivo y se probó en la estación de trabajo para nuevamente realizar Slos estudios ergonómicos y determinar el impacto de la propuesta. La tabla 5 resume los datos obtenidos de los análisis REBA y owAs, así como su comparación con el método de trabajo anterior.

Tabla 5. Comparativa de análisis REBA y owAs del proceso de acabado

\begin{tabular}{lcccc}
\hline \multirow{2}{*}{ Actividad } & \multicolumn{2}{c}{ REBA } & \multicolumn{2}{c}{ owAs } \\
\cline { 2 - 5 } & $\begin{array}{c}\text { Puntaje } \\
\text { anterior }\end{array}$ & $\begin{array}{c}\text { Puntaje pro- } \\
\text { puesta imple- } \\
\text { mentada }\end{array}$ & $\begin{array}{c}\text { Categoría } \\
\text { de acción } \\
\text { anterior }\end{array}$ & $\begin{array}{c}\text { Categoría de acción con } \\
\text { propuesta implemen- } \\
\text { tada }\end{array}$ \\
\hline Sujeción & 11 & 3 & 4 & 1 \\
Colocación & 12 & 8 & 2 & 1 \\
Acomodo & 12 & 7 & 4 & 3 \\
\hline
\end{tabular}

Implementado el movimiento de empujar la carga actual al punto deseado genera un total de energía consumida igual a $3032 \mathrm{kcal}$ en un periodo de $8908 \mathrm{~s}$ con una tasa promedio de $20.4 \mathrm{kcal} / \mathrm{min}$. 


\section{Discusión}

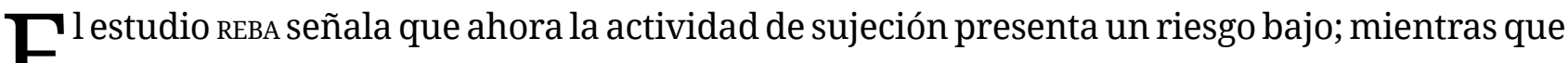
Elas actividades de colocación y acomodo se encuentran en un punto medio y que requieren más acciones para disminuir los riesgos ergonómicos. Así, los hallazgos del análisis REBA de esta investigación ratifican los encontrados en trabajadores de la industria del caucho y en mecánicos automotores en Irán $(11,12)$.

Por su parte, el análisis owAs destaca que las actividades de sujeción y colocación del componente automotor tienen una postura normal y natural, sin efectos dañinos en el sistema músculo-esquelético, mismo caso al presentado en el estudio de cargas ergonómicas efectuado a tres contadores en su lugar de trabajo (13). Sin embargo, la operación de acomodo sí requiere acciones correctivas inmediatas.

Una vez implementado el dispositivo en el centro de trabajo, las actividades de sujeción y colocación del guarda fango quedan en una categoría de acción 1 , similares a las observadas en posturas adoptadas con mayor frecuencia por los instrumentadores durante las cirugías (14). Relacionado con la actividad de colocación, cuya categoría de acción inicial fue de 2, el estudio a los administradores del catering muestra que las posturas podrían causar daño al sistema músculo-esquelético, por lo cual se deben tomar acciones correctivas a corto plazo (15).

Con respecto a los estudios de NIosh, el dispositivo evitó que se realice la carga por parte de la trabajadora y el índice levantamiento en el origen pasó de un valor de 1.46 a 0.64. Por su parte, dicho índice en el destino se modificó de 0.85 a un valor de 0.88 , lo cual implica que el trabajo lo pueden realizar la mayoría de los trabajadores. Según estos resultados, en el proceso de acabado (origen) existe una alarma respecto al peso de la carga manipulada; sin embargo, es preciso destacar que en México existe un problema de obesidad severa entre la población y que este aspecto no fue considerado en los estudios con las trabajadoras del área de producción, situación que se resalta, al mostrar que la población con obesidad severa no está dentro de la cobertura de la ecuación de Niosh (16). Tampoco se consideró el hecho de una fuerza laboral envejecida y con capacidad de elevación disminuida (17).

El método de indicadores clave presenta una puntuación del riesgo igual a 24. Ello significa que se trata de una situación de aumento de la carga y que, probablemente, sobrecargue a trabajadores con poca resistencia; se sugiere, entonces, un rediseño del proceso de acabado. Este análisis comprobó los resultados del método кім, que plantea altas calificaciones en lugares de trabajo con demasiadas operaciones de manejo manual y altas frecuencias de síntomas músculo-esqueléticos en trabajadores expuestos (18).

Además, el trabajo de investigación combina técnicas ergonómicas, en este caso REBA, oWAS, NIOSH Y KIM, reconocidas para determinar posibles riesgos laborales al efectuar tareas encomendadas, tal y como se verificó en el estudio del uso de dispositivos de medición en procesos de inspección de calidad en el sector automotor (19). 
El presente trabajo demostró que instrumentos ergonómicos como REBA, NIOSH y el análisis de las condiciones de trabajo contribuyen a evaluar las posturas de trabajo y problemas de repetibilidad, debido a la naturaleza de la tarea, tal y como se confirmó en estudios similares en el sector de la maricultura (20). A su vez, se comprobó que las actividades de manejo de materiales (como levantamiento, bajando, empujando, tirando y cargando) inducen riesgos físicos entre los obreros de las fábricas, por ejemplo (21).

Los autores reconocen que existen más opciones para disminuir los riesgos posturales en esta actividad del sector automotor que no se consideraron, por lo que en futuras investigaciones se centrarán en el desarrollo de controles de ingeniería y administrativos (22).

\section{Contribución de los autores}

【 a concepción y el diseño del artículo, así como la planeación y desarrollo de contenido, Lcorrieron a cargo de los tres autores. La versión final para publicar fue elaborada por el Dr. Luis Cuautle y el Mtro. Luis Alberto Uribe.

\section{Conflicto de intereses}

Ninguno declarado.

\section{Descargos de responsabilidad}

S e protegió la vida, la salud, la dignidad, la integridad, el derecho a la autodeterminación, la intimidad y la confidencialidad de la información personal de quienes participaron en la investigación.

\section{Referencias}

1. Kandananond $\mathrm{K}$. The incorporation of virtual ergonomics to improve the occupational safety condition in a factory. Int J Metrol Qual Eng. 2018;9(4):1-9. https://doi.org/10.1051/ ijmqe/2018013

2. Secretaría de Salud Laboral de ccoo de Madrid. Métodos de evaluación ergonómica [internet]. Madrid: Comisiones Obreras de Madrid; 2016. Disponible en: http://www. saludlaboralmadrid.es/portfolio-item/metodos-de-evaluacion-ergonomica/ 
3. Araújo AV, Arcanjo GM, Fernandes H, Arcanjo GS. Ergonomic work analysis: a case study of bus drivers in the private collective transportation sector. Work. 2018;60:41-47. https:// doi.org/10.3233/WOR-182718

4. Ahmadi S, Zare S, Ghanbari R, Bahrami S, Zaroushani V. The effect of increased manufacturing rate on risk of low back pain. J Paramed Sci. 2018:9(3):49-55. https://doi. org/10.22037/JPS.V9I3.18466

5. International Organization for Standardization. Iso11226: ergonomics-evaluation of static working postures. Tokio; 2000.

6. International Organization for Standardization. Iso11228-1: ergonomics-manual handling-part 1: lifting and carrying. Tokio; 2007.

7. International Organization for Standardization. Ergonomics-manual handling-part 2: pushing and pulling. Tokio; 2007.

8. Micheletti M, Giustetto A, Caffaro F, Colantoni A, Cavallo E, Grigolato S. Risk assessment for musculoskeletal disorders in forestry: a comparison between RULA and REBA in the manual feeding of a wood-chipper. Int J Environ Res Public Health. 2019;16(5):793. https://doi.org/10.3390/ijerph16050793

9. Valdenebro Olea L, López Acosta M, Quirós Morales AF, Montiel Rodríguez LC, Sánchez Padilla JE. Evaluación ergonómica de un puesto de trabajo en el sector metalmecánico. Rev Ing Industrial. 2016;15(1):69-83.

10. Klussmann A, Liebers F, Gebhardt H, Rieger MA, Latza U, Steinberg U. Risk assessment of manual handling operations at work with the key indicator method (кім-мно): determination of criterion validity regarding the prevalence of musculoskeletal symptoms and clinical conditions within a cross-sectional study. BMC Musculoskelet Disord. 2017;18(1):184. https://doi.org/10.1186/s12891-017-1542-0

11. Samaei SE, Tirgar A, Khanjani N, Mostafaee M, Hossinabadi MB. Effect of personal risk factors on the prevalence rate of musculoskeletal disorders among workers of an Iranian rubber factory. Work. 2017;57(4):547-53. https://doi.org/10.3233/WOR-172586

12. Moradi M, Poursadeghiyan M, Khammar A, Hami M, Darsnj A, Yormohammadi H. REBA method for the ergonomic risk assessment of auto mechanics postural stress caused by working conditions in Keranshah (Iran). Ann trop Med Public Health. 2017;10(3):289-94. https://doi.org/10.4103/ATMPH.ATMPH_107_17

13. Kacjan K, Govekar K, Oder M. Ergonomic burdens and harms at the workplace of accountants. Int J Sanitary Eng Res [internet]. 2012;10(1):61-72. Disponible en: https://journal. institut-isi.si/wp-content/uploads/2017/01/SI-110-2016-Kacjan-Zgajnar-Govekar-Oder.pdf

14. Velasco-Rey KM. Análisis de las posturas adoptadas por instrumentadores quirúrgicos durante cirugía. Rev Salud Publica. 2017;19(1):112-7. http://dx.doi.org/10.15446/rsap. v19n1.36473

15. Molina R, Galarza I, Villegas C, López P. Evaluación de riesgos ergonómicos del trabajo en empresas de catering. Turismo y Sociedad. 2018;23:101-23. https://doi. org/10.18601/01207555.n23.06

16. Singh D, Park W, Hwang D, Levy M.S. Severe obesity effect on low back biomechanical stress of manual load lifting. Work. 2015;51:337-48. https://doi.org/10.3233/WOR-141945 
17. Markiewicz D. How to reduce heavy lifting: try this "soft sell" approach. Industrial and Hygiene. 2017;5(7).

18. KluBmann A, Gebhardt H, Rieger M, Liebers F, Steinberg U. Evaluation of objectivity, reliability and criterion validity of the Key Indicator Method for Manual Handling Operations (кIM-мHо), draft 2007. Work;2012;41(1):3997-4003. https://doi.org/10.3233/ WOR-2012-0699-3997

19. Cuautle-Gutiérrez L, Escobedo-Portillo M, Uribe-Pacheco L, García-Tepox J. Medición del riesgo de lesión de espalda en trabajadores mexicanos. Rev Cienc Salud. 2019;17(2): 175-87. http://doi.org/10.12804/revistas.urosario.edu.co/revsalud/a.7921

20. Peck GM, Guertler C, Quadros W, García L, Díaz EA. Work ergonomic analysis: application of a postural study on the oysters cultivation. J Health Sci. 2019;21(1):15-20. http:// doi.org/10.17921/2447-8938.2019v21n1p15-20

21. Deros B, Daruis D, Basir I. A study on ergonomic awareness among workers performing manual material handling activities. Procedia Soc Behav Sci. 2015;195:1666-73. https:// doi.org/10.1016/j.sbspro.2015.06.238

22. Fathallah FA. Musculoskeletal disorders in labor-intensive agriculture. Applied Ergon. 2010;41(6):738-43. https://doi.org/10.1016/j.apergo.2010.03.003 